PROCEEDINGS OF THE

AMERICAN MATHEMATICAL SOCIETY

Volume 135, Number 2, February 2007, Pages 313-328

S 0002-9939(06)08353-5

Article electronically published on September 11, 2006

\title{
EQUILIBRIUM POINT OF GREEN'S FUNCTION FOR THE ANNULUS AND EISENSTEIN SERIES
}

\author{
AHMED SEBBAR AND THÉRÈSE FALLIERO
}

(Communicated by Richard A. Wentworth)

\begin{abstract}
We study the motion of the equilibrium point of Green's function and give an explicit parametrization of the unique zero of the Bergman kernel of the annulus. This problem is reduced to solving the equation $\wp(z, \tau)=$ $-\frac{\pi^{2}}{3} E_{2}(\tau)$, where $E_{2}(\tau)$ is the usual Eisenstein series.
\end{abstract}

\section{INTRODUCTION}

In 2, 3], Eichler and Zagier gave an explicit formula for the solution $z_{0}(\tau)$ for $\Im \tau>0$ of the equation $\wp(z, \tau)=0, \wp$ being the Weierstrass function of periods 1 and $\tau$. They also observe that the method developed can be applied to find the solution to any equation of the form $\wp(z, \tau)=\phi(\tau)$, where $\phi$ is a modular form of weight 2 . In this work, we solve the equation $\wp(z, \tau)=-\frac{\pi^{2}}{3} E_{2}(\tau)$, where $E_{2}(\tau)$ is an Eisenstein series which is not a modular form. One motivation for studying such an equation is that a close connection is established between equilibrium points (critical points) of Green's functions of the annulus and Eisenstein series. The links between the apparently distant themes rely on the role played by the Green's function for multiply connected domains, their equilibrium points with their motions, and by classical kernel functions such as the Bergman kernel or the Schiffer kernel on Riemann surfaces. This connection brings together concepts from analysis, geometry and number theory.

More precisely, our main result (Theorem 3.2) is the following: if $C_{R}=\{R<$ $|z|<1\}$ is a given annulus, as the pole of the Green's function of $C_{R}$ goes to the boundary, the limiting position of the equilibrium point is given by an equation of the form $\wp(z, \tau)=-\frac{\pi^{2}}{3} E_{2}(\tau)$. We will explain how the equilibrium point depends on the Bergman kernel of the annulus $C_{R}$. In particular, the zeros of the Bergman kernel will exhibit, for a given $R>1$, two real numbers $R_{1}, R_{2} \in(1, R)$ with $R_{1} R_{2}=R$ and such that the annulus $\left\{R_{1}<|z|<R_{2}\right\}$ contains all the equilibrium points of the Green's function of $C_{R}$. The important fact here is that the radii of the two annulus have equal geometrical means. We recover in an effective manner the results of Maria [10] and complete a result of Rudin [12.

Received by the editors January 25, 2005 and, in revised form, June 1, 2005.

2000 Mathematics Subject Classification. Primary 11F03, 11F11, 30C40, 34B30.

Key words and phrases. Equilibrium points, Eisenstein series, Bergman kernel.

We are grateful to Henri Cohen and Don Zagier for teaching us some facts about the zeros of the Eisenstein series $E_{2}$. 
A second motivation for studying the equation $\wp(z, \tau)=-\frac{\pi^{2}}{3} E_{2}(\tau)$ relies on an important theorem of Schiffer and Hawley [14] and also Hejhal [7]: For a bounded plane domain $D$ with analytic boundary $\partial D$ and connectivity $1 \leq p \leq \infty$, there exists a relationship between the curvature of the Bergman metric, the Schiffer kernels and their zeros. Finally, a physical interpretation may be given to the notion of equilibrium point; if the boundary of $C_{R}$ is kept at potential zero (the potential at an arbitrary point $W \in C_{R}$ is due to a unit charge at a fixed $Z \in C_{R}$ and the charge on the boundary induced by it is precisely $G(Z, W)$ ), the equilibrium point is the point where the intensity of the corresponding field of force is zero. It seems of interest to study the link between this physical picture, Eisenstein series, the Bergman kernel and the Bergman metric in higher connectivity.

\section{Statement of the Problem}

Let $D_{p}$ be a bounded open set of the complex plane $\mathbb{C}$ bordered by $p$ analytic curves $\Gamma_{1}, \Gamma_{2}, \cdots, \Gamma_{p-1}$ and an outer boundary $\Gamma_{0}$. By the classical Hopf's lemma, the normal derivative of the the Dirichlet Green's function is positive on the boundary $\Gamma_{0} \cup \Gamma_{1} \cup \cdots \cup \Gamma_{p-1}$ of $D_{p}$, and one may ask if there is a compact set $\mathcal{K}$ in $D_{p}$, independent of $z_{0}$, containing all the equilibrium points of the (complex) Green's function $G\left(z_{0}, z\right)$ of $D_{p}$. Our objective in this work is to give a full answer in the case $p=1$. The same question should be asked in $\widehat{\mathbb{C}} \backslash E$, where $E$ is a given union of intervals $E=\left[E_{1}, E_{2}\right] \cup\left[E_{3}, E_{4}\right] \cup \cdots \cup\left[E_{2 n-1}, E_{2 n}\right]$. In fact a theorem of Koebe asserts that $\widehat{\mathbb{C}} \backslash E$ can be mapped conformally into a domain $D_{n}$ and such that each component $\Gamma_{i}$ is a circle, $0 \leq i \leq n-1$. On the other hand, the complex Green's function (analytic completion of the classical Green's function) of $\widehat{\mathbb{C}} \backslash E$ with a pole at $x_{0}$ is given by the abelian integral [17] (p. 227):

$$
G\left(x_{0}, z\right)=\int_{E_{2 n}}^{z} h(\zeta) q(\zeta)^{-\frac{1}{2}} \frac{1}{\zeta-x_{0}} d \zeta
$$

where $q(\zeta)=\left(\zeta-E_{1}\right)\left(\zeta-E_{2}\right) \cdots\left(\zeta-E_{2 n}\right)$ and $h(\zeta)$ is a monic polynomial of degree $n-1, h(\zeta)=\zeta^{n-1}+h_{n-2} \zeta^{n-2}+\cdots+h_{0}$ with coefficients satisfying the following system of the Jacobi inversion problem:

$$
\sum_{0 \leq k \leq n-1} h_{k} \int_{E_{2 j}}^{E_{2 j+1}} \zeta^{k}|q(\zeta)|^{-\frac{1}{2}} \frac{d \zeta}{\zeta-x_{0}}=0, \quad 1 \leq j \leq n-1,
$$

and

$$
\sum_{0 \leq k \leq n-1} h_{k} x_{0}^{k}=-q\left(x_{0}\right)^{\frac{1}{2}}
$$

If $x_{0}=\infty$, the Green's function of $\widehat{\mathbb{C}} \backslash E$ with a pole at $\infty$ is now given by

$$
G(z)=\int_{E_{2 n}}^{z} h(\zeta) q(\zeta)^{-\frac{1}{2}} d \zeta
$$

and the system

$$
\sum_{0 \leq k \leq n-1} h_{k} \int_{E_{2 j}}^{E_{2 j+1}} \zeta^{k}|q(\zeta)|^{-\frac{1}{2}} d \zeta=0, \quad 1 \leq j \leq n-1 ; \quad h_{n-1}=1 .
$$


Definition 2.1. The equilibrium (or critical) points of the Green's function with pole at a finite point $x_{0}$ are the zeros $c_{1}=c_{1}\left(x_{0}\right), c_{2}=c_{2}\left(x_{0}\right), \cdots, c_{n-1}=c_{n-1}\left(x_{0}\right)$ of the polynomial $h(\zeta)=h_{n-1} \zeta^{n-1}+h_{n-2} \zeta^{n-2}+\cdots+h_{0}$, with the above conditions (2.2), (2.3).

For $x_{0}=\infty$, the equilibrium points $c_{i}, 1 \leq i \leq n-1$, are all real and simple, located in the gaps $\left(E_{2 j}, E_{2 j+1}\right), 1 \leq j \leq n-1$. Furthermore, their images are given by [17]:

$$
G\left(c_{j}\right)=\frac{1}{2} \int_{E_{2 j}}^{E_{2 j+1}}|h(\zeta)| q(\zeta)^{-\frac{1}{2}} d \zeta .
$$

From now on, we consider only the case of two intervals and without any loss of generality we take $E=[-1, \alpha] \cup[\beta, 1],-1<\alpha<0<\beta<1$. In this case, the Green's function of $\widehat{\mathbb{C}} \backslash E$ with pole $x_{0}$ is

$$
G\left(x_{0}, z\right)=\int_{1}^{z} \frac{k(\zeta)}{q(\zeta)^{\frac{1}{2}}} \frac{d \zeta}{\zeta-x_{0}}
$$

with $q(\zeta)=\left(\zeta^{2}-1\right)(\zeta-\alpha)(\zeta-\beta)$, and $k(\zeta)=k_{0}+k_{1} \zeta$ is a polynomial with complex coefficients determined by the two conditions:

$$
\int_{\alpha}^{\beta} \frac{k(\zeta)}{q(\zeta)^{\frac{1}{2}}} \frac{d \zeta}{\zeta-x_{0}}=0, \quad k\left(x_{0}\right)=-q\left(x_{0}\right)^{\frac{1}{2}}
$$

The next result is an immediate consequence of the definition of the equilibrium point $c\left(x_{0}\right)$.

Proposition 2.2. Let $\rho=\max (|\alpha|,|\beta|)$ and define the sequence of moments $a_{n}=$ $\int_{\alpha}^{\beta} \frac{\zeta^{n}}{q(\zeta)^{\frac{1}{2}}} d \zeta$. Then for $\left|x_{0}\right|>\rho$ the equilibrium point is given by

$$
c\left(x_{0}\right)=\frac{\sum_{n \geq 1} a_{n} x_{0}^{-n}}{\sum_{n \geq 1} a_{n-1} x_{0}^{-n}}
$$

in particular

$$
c(\infty)=\frac{\int_{\alpha}^{\beta} \frac{\zeta}{q(\zeta)^{\frac{1}{2}}} d \zeta}{\int_{\alpha}^{\beta} \frac{1}{q(\zeta)^{\frac{1}{2}}} d \zeta} .
$$

This is easily obtained from the representation of $c\left(x_{0}\right)$ as a quotient of two transforms of Cauchy type:

$$
c\left(x_{0}\right)=\frac{\int_{\alpha}^{\beta} \frac{\zeta}{q(\zeta)^{\frac{1}{2}}} \frac{d \zeta}{\zeta-x_{0}}}{\int_{\alpha}^{\beta} \frac{1}{q(\zeta)^{\frac{1}{2}}} \frac{d \zeta}{\zeta-x_{0}}} .
$$

The function $x_{0} \mapsto c\left(x_{0}\right)$ is an analytic function of $x_{0}$ on the simply connected domain $\widehat{\mathbb{C}} \backslash[\alpha, \beta]$, and it can be given a Laurent expansion in the neighborhood of infinity in $\widehat{\mathbb{C}}$,

$$
c\left(x_{0}\right)=\sum_{n \geq 0} A_{n} x_{0}^{-n}, \quad A_{n}=a_{0}^{-(n+1)} H_{n}\left(a_{0}, a_{1}, \cdots, a_{n+1}\right), \quad\left|x_{0}\right|>\rho,
$$


where $H_{0}\left(a_{0}, a_{1}\right)=a_{1}$ and for $n \geq 1$ :

$$
H_{n}\left(a_{0}, a_{1}, \cdots, a_{n+1}\right)=\left|\begin{array}{cccccc}
a_{0} & 0 & & & & a_{1} \\
a_{1} & a_{0} & 0 & & & a_{2} \\
& a_{1} & \ddots & & & \\
& & & & & \\
& & & \ddots & \ddots & \\
& & & \ddots & \ddots & a_{n} \\
& & & & a_{1} & a_{n+1}
\end{array}\right| .
$$

The determinant $H_{n}\left(a_{0}, a_{1}, \cdots, a_{n+1}\right)$ is a homogeneous polynomial of degree $n+1$, $H_{1}\left(a_{0}, a_{1}, a_{2}\right)=a_{0} a_{2}-a_{1}^{2}, H_{2}\left(a_{0}, a_{1}, a_{2}, a_{3}\right)=a_{0}^{2} a_{3}-2 a_{0} a_{1} a_{2}+a_{1}^{3}, \cdots$. They are actually related to Faber polynomials. For a formal Laurent series

$$
g(t)=b t+b_{0}+\frac{b_{1}}{t}+\cdots, b \neq 0,
$$

the Faber polynomials $\Phi_{n}(w)$ are defined by

$$
\log \frac{g(t)-w}{b t}=-\sum_{n=1}^{\infty} \frac{\Phi_{n}(w)}{n} t^{-n} .
$$

If we take the derivative in $w$ and choose $b=1, b_{k}=\frac{a_{k+1}}{a_{0}}, k \geq 0$, we find from (2.6) that

$$
A_{n}=-\frac{\Phi_{n+2}^{\prime}(0)}{n+2} .
$$

Now, we would like to take up the problem of the variation of the function $c\left(x_{0}\right)$ from the annulus model point of view. We conformally map the region $\widehat{\mathbb{C}} \backslash[-1, \alpha] \cup[\beta, 1]$ on the annulus $A_{r, 1}=\{r<|v|<1\}, r=e^{-\pi K / K^{\prime}}$. The point at infinity $\infty$ is sent onto a point $s$ of the positive real half line. This conformal map is given by ([1], p. $282 ;$ 4])

$$
z=\frac{c n^{2} M s n^{2} u+s n^{2} M c n^{2} u}{s n^{2} u-s n^{2} M}, \quad z \in \widehat{\mathbb{C}} \backslash[-1, \alpha] \cup[\beta, 1],
$$

with classical notations for

$$
w=\int_{0}^{x} \frac{d x}{\sqrt{\left(1-x^{2}\right)\left(1-k^{2} x^{2}\right)}}, x=s n w
$$

and

$$
\begin{aligned}
k^{2} & =\frac{2(\beta-\alpha)}{(1+\beta)(1-\alpha)} \in(0,1), \quad k^{\prime 2}=1-k^{2}, \\
K & =\int_{0}^{1} \frac{d x}{\sqrt{\left(1-x^{2}\right)\left(1-k^{2} x^{2}\right)}}, \quad K^{\prime}=\int_{0}^{1} \frac{d x}{\sqrt{\left(1-x^{2}\right)\left(1-k^{\prime 2} x^{2}\right)}}, \\
M & =\int_{0}^{\sqrt{\frac{1-\alpha}{2}}} \frac{d t}{\sqrt{\left(1-t^{2}\right)\left(1-k^{2} t^{2}\right)}}=\frac{K^{\prime} \log s}{\pi} \\
\tau & =i K^{\prime} / K, \quad r=e^{-i \pi / \tau}, u=\frac{K^{\prime} \log v}{\pi}
\end{aligned}
$$


and the Jacobi theta functions which will be used later:

$$
\begin{aligned}
\theta_{1}(z, \tau) & =2 \sum_{n=0}^{\infty}(-1)^{n} e^{i \pi \tau(n+1 / 2)^{2}} \sin (2 n+1) \pi z, \\
\theta_{2}(z, \tau) & =2 \sum_{n=0}^{\infty} e^{i \pi \tau(n+1 / 2)^{2}} \cos (2 n+1) \pi z, \\
\theta_{3}(z, \tau) & =1+2 \sum_{n=1}^{\infty} e^{i \pi \tau n^{2}} \cos 2 n \pi z \\
\theta_{4}(z, \tau) & =1+2 \sum_{n=1}^{\infty}(-1)^{n} e^{i \pi \tau n^{2}} \cos 2 n \pi z, \\
\text { snu } & =\frac{1}{\sqrt{k}} \frac{\theta_{1}(u)}{\theta_{4}(u)}, \quad c n^{2} u=1-s n^{2} u .
\end{aligned}
$$

Therefore the Green's function of $\widehat{\mathbb{C}} \backslash([-1, \alpha] \cup[\beta, 1])$ has the form

$$
G(x)=G(x, \infty)=-\log \left|\frac{\theta_{1}\left(\frac{u-M}{2 K}, \tau\right)}{\theta_{1}\left(\frac{u+M}{2 K}, \tau\right)}\right| .
$$

On the other hand, the Green's function of the annulus $A_{r, 1}=\{r<|v|<1\}, 0<$ $r<1$, can also be given by (see [4 and the references therein)

$$
G(z, w)=\Re \log \frac{w^{1 / 2} z^{-\log w / 2 \log r}(1-z / w) \prod_{m=1}^{\infty}\left(1-r^{2 m} z / w\right)\left(1-r^{2 m} w / z\right)}{\bar{w}^{-1 / 2} z^{\log \bar{w} / 2 \log r}(1-z \bar{w}) \prod_{m=1}^{\infty}\left(1-r^{2 m} z \bar{w}\right)\left(1-r^{2 m} / z \bar{w}\right)} .
$$

In the next section, we derive closed expressions for Green's functions and prove our main result.

\section{A formula for the zero of the Bergman Kernel}

We recall some essential facts concerning the Bergman kernel that we need for our investigation on the motion of the equilibrium point. For a finite not necessarily simply connected open Riemann surface $\Omega$, the Bergman kernel of $\Omega$, denoted by $K(z, \bar{t}) d z d \bar{t}$, is the kernel characterized by the reproducing property for holomorphic differentials $\alpha(t) d t$, where

$$
\alpha(z)=\frac{1}{2 i} \iint_{\Omega} K(z, \bar{t}) d \bar{t} \wedge \alpha(t) d t .
$$

It has been shown by Schiffer [13] that the Bergman kernel is given by the second derivative of the Green's function:

$$
K(z, \bar{t})=-\frac{2}{\pi} \frac{\partial^{2} G(z, t)}{\partial z \partial \bar{t}} .
$$

The adjoint kernel or the Schiffer kernel is defined by

$$
L(z, t)=-\frac{2}{\pi} \frac{\partial^{2} G(z, t)}{\partial z \partial t}
$$

and we have the important relation [6] and [15]

$$
K(z, \bar{t}) d z=-L(z, t) d z, \quad z \in \Omega, t \in \partial \Omega .
$$

In their answer to the Lu Qi-keng conjecture for finite Riemann surfaces, Suita and Yamada [15] proved that for a finite Riemann surface $\Omega$ which is not simply 
connected, the Bergman kernel $K(z, \bar{t})$ has exactly $n+2 p-1$ zeros as a function of $z$, for $t \in \partial \Omega$ and for $n$ and $p$ being respectively the number of boundary contours and the genus of $\Omega$. In particular, if $\Omega$ is a plane domain with two boundary components, the Bergman kernel is a function and has only one zero. Our objective is to give a complete description of this zero by using some results on Jacobi forms. On the other hand, Zarankiewicz in 18 and Fay in 5. (p. 133) gave an expression of the kernel-function for the annulus $A_{r}=\{r<|z|<1\}$ in a closed form

$$
K(z, \bar{t})=\frac{1}{\pi z \bar{t}}\left\{\wp\left(\log (z \bar{t}) ; 2 \log \frac{1}{r}, 2 i \pi\right)+\frac{\zeta\left(i \pi ; 2 \log \frac{1}{r}, 2 i \pi\right)}{i \pi}\right\}
$$

or

$$
K(z, \bar{t})=\frac{1}{\pi z \bar{t}}\left\{\wp\left(\log (z \bar{t}) ; 2 \omega, 2 \omega^{\prime}\right)+\frac{\eta}{\omega}-\frac{1}{2 \omega^{\prime}}\right\},
$$

where $\wp$ and $\zeta$ are the Weierstrass functions determined by the periods $2 \omega=2 i \pi$ and $2 \omega^{\prime}=2 \log r$. From (2.8) and (3.2) we obtain at once:

Proposition 3.1. For $z, t \in A_{r}, z \neq w$,

$$
K(z, \bar{t})=\frac{1}{|t|^{2}} L\left(z, \frac{1}{t}\right) .
$$

In particular the Schiffer kernel has no zero in $A_{r}$.

In order to formulate the result on the parametrization of the zero of the kernel function of the annulus, let us introduce the classical Eisenstein series and other arithmetical functions:

$$
\begin{aligned}
& E_{2}(\tau)=1-24 \sum_{n=1}^{\infty} \frac{n q^{n}}{1-q^{n}}=1-24 \sum_{n=1}^{\infty} \sigma_{1}(n) q^{n} \\
& E_{4}(\tau)=1+240 \sum_{n=1}^{\infty} \frac{n^{3} q^{n}}{1-q^{n}}=1+240 \sum_{n=1}^{\infty} \sigma_{3}(n) q^{n} \\
& E_{6}(\tau)=1-504 \sum_{n=1}^{\infty} \frac{n^{5} q^{n}}{1-q^{n}}=1-504 \sum_{n=1}^{\infty} \sigma_{5}(n) q^{n}
\end{aligned}
$$

with

$$
\sigma_{k}(n)=\sum_{d \mid n} d^{k}, \quad q=e^{2 i \pi \tau}
$$

It is well known that $E_{4}(\tau)$ and $E_{6}(\tau)$ are modular forms of weights 4 and 6 for the group $\Gamma=\mathrm{SL}_{2}(\mathbb{Z})$. $E_{2}(\tau)$ is not modular, but

$$
E_{2}^{*}(\tau)=1-24 \sum_{n=1}^{\infty} \frac{n q^{n}}{1-q^{n}}-\frac{3}{\pi \Im \tau}=1-\frac{3}{\pi \Im \tau}-24 q-72 q^{2}-96 q^{3}-168 q^{4}-\cdots
$$

is the non-holomorphic Eisenstein series of weight 2 for $\Gamma$. We recall the classical discriminant function $\Delta$ defined by the infinite product

$$
\Delta(\tau)=q \prod_{n=1}^{\infty}\left(1-q^{n}\right)^{24}=q-24 q^{2}+252 q^{3}-1472 q^{4}+\cdots
$$


Then $\Delta(\tau)$ is a cusp form for $\Gamma$ of weight 12 and verifies the relation $1728 \Delta=$ $E_{4}^{3}-E_{6}^{2}$. We introduce, following Ramanujan, the functions

$$
\Phi_{r, s}(q)=\sum_{m=1}^{m=\infty} \sum_{n=1}^{n=\infty} m^{r} n^{s} q^{m n}
$$

We refer to [1] for the following properties of these functions:

$$
\begin{aligned}
\Phi_{r, s}(q) & =\Phi_{s, r}(q), \\
\Phi_{r, s}(q) & =\left(q \frac{d}{d q}\right)^{r} \Phi_{0, s-r}(q) .
\end{aligned}
$$

Sometimes, we simply write $\Phi_{r, s}(\tau)$ for $\Phi_{r, s}(q)$ and we have the algebraic relation

$$
\Phi_{r, s}(\tau)=\sum_{\substack{2 l+4 m+6 n=r+s+1 \\ l-1 \leq \inf (r, s)}} K_{l, m, n} E_{2}(\tau)^{l} E_{4}(\tau)^{m} E_{6}(\tau)^{n} .
$$

In the sequel, we consider new half-periods $\omega_{1}, \omega_{3}$ with $\omega_{1}=-\omega^{\prime}=\log \frac{1}{r}=\log R$ and $\omega_{3}=\omega=i \pi$.

Theorem 3.2. For each $R>1$ and for each $Z \in C_{R}=\{1<|Z|<R\}$, the Bergman kernel $K(Z, \bar{W})$, as a function of $W$, has a unique zero $W=W(Z)$ in $C_{R}$ given by

$$
\wp(\log (Z \bar{W}) ; 2 \log R, 2 i \pi)=\frac{1}{\left(2 \omega_{1}\right)^{2}} \phi(\tau), \quad \phi(\tau)=-\frac{\pi^{2}}{3} E_{2}(\tau), \quad \tau=\frac{i \pi}{\log R} .
$$

Moreover, $u(\tau)=\frac{1}{2 \omega_{1}} \log (Z \bar{W})$ has the following integral representation modulo $\mathbb{Z}+\tau \mathbb{Z}:$

$$
u(\tau)= \pm \frac{\tau}{2} \pm \frac{3}{4} \pm \frac{1728 \sqrt{3}}{5} \pi \int_{\tau}^{i \infty} \frac{\Phi_{5,6}(t)-\Delta(t)}{\Phi_{2,3}(t)^{\frac{3}{2}}}(t-\tau) d t .
$$

In particular $\arg Z-\arg W(Z)=\pi$ for every $Z \in C_{R}$.

Proof. Let us rewrite equation (3.4) in a convenient form for our calculations, which will also explain the apparition of the Eisenstein series $E_{2}$. From the Legendre relation $\eta \omega^{\prime}-\eta^{\prime} \omega=i \frac{\pi}{2}$, (3.4) can be simply written as

$$
K(z, \bar{t})=\frac{1}{\pi z \bar{t}}\left\{\wp\left(\log (z \bar{t}) ; 2 \omega, 2 \omega^{\prime}\right)+\frac{\eta^{\prime}}{\omega^{\prime}}\right\} .
$$

If $R=1 / r$, the holomorphic involution $i: z \mapsto Z=1 / z$ transforms the annulus $A_{r}$ into the annulus $C_{R}=\{1<|z|<R\}$, and the kernel functions of the two domains are related by the identity

$$
K_{A_{r}}(z, \bar{w})=K_{C_{R}}(Z, \bar{W}) i^{\prime}(z) \overline{i^{\prime}(w)} .
$$

In particular we obtain that

$$
K_{C_{R}}(Z, \bar{W})=\frac{1}{\pi Z \bar{W}}\left\{\wp\left(\log (Z \bar{W}) ; 2 \omega, 2 \omega^{\prime}\right)+\frac{\eta^{\prime}}{\omega^{\prime}}\right\} .
$$

Now, $\wp\left(u ; 2 \omega, 2 \omega^{\prime}\right)=\wp\left(u ; 2 \omega_{1}, 2 \omega_{3}\right)$ and in general, if $\omega, \omega^{\prime}$ are given with $\Im \frac{\omega^{\prime}}{\omega}>0$ and if

$$
\left(\begin{array}{l}
\omega_{1} \\
\omega_{3}
\end{array}\right)=\left(\begin{array}{ll}
\alpha & \beta \\
\gamma & \delta
\end{array}\right)\left(\begin{array}{c}
\omega \\
\omega^{\prime}
\end{array}\right)
$$


with $\left(\begin{array}{cc}\alpha & \beta \\ \gamma & \delta\end{array}\right) \in \mathrm{SL}_{2}(\mathbb{Z})$, the periods undergo the same transformation as $\omega, \omega^{\prime}$ and

$$
\frac{\eta}{\omega}-\frac{\eta_{1}}{\omega_{1}}=\frac{\eta}{\omega}-\frac{\alpha \eta+\beta \eta^{\prime}}{\alpha \omega+\beta \omega^{\prime}}=\frac{\beta\left(\eta \omega^{\prime}-\eta^{\prime} \omega\right)}{\omega \omega_{1}}=\frac{\beta \pi i}{2 \omega \omega_{1}} .
$$

In our case, the period of the Weierstrass $\zeta$-function transforms as

$$
\frac{\eta_{1}}{2 \omega_{1}}=\frac{\eta}{2 \omega}-\frac{i \pi}{4 \omega \omega^{\prime}}=\frac{\eta}{2 \omega}-\frac{1}{4 \omega^{\prime}} .
$$

We will write $\tau=-\frac{\omega}{\omega^{\prime}}=\frac{\omega_{3}}{\omega_{1}}$, hence $\wp\left(u ; 2 \omega, 2 \omega^{\prime}\right)+\frac{\eta}{\omega}-\frac{1}{2 \omega^{\prime}}=\wp\left(u ; 2 \omega_{1}, 2 \omega_{3}\right)+\frac{\eta_{1}}{\omega_{1}}$, and substituting into (3.4), we get the more convenient formula

$$
K_{C_{R}}(Z, \bar{W})=\frac{1}{\pi Z \bar{W}}\left\{\wp\left(\log (Z \bar{W}) ; 2 \omega_{1}, 2 \omega_{3}\right)+\frac{\eta_{1}}{\omega_{1}}\right\}
$$

The Eisenstein series $E_{2}$ appears when we make explicit the local behavior of $\wp\left(u ; 2 \omega_{1}, 2 \omega_{3}\right)$ from its Fourier expansion. Indeed the function $u \mapsto \wp\left(u ; 2 \omega_{1}, 2 \omega_{3}\right)$ is an even function with pole of order two at the origin. The development at $u=0$ does not contain a constant term and can be obtained for instance from the Fourier series

$$
\begin{aligned}
& \wp\left(u ; 2 \omega_{1}, 2 \omega_{3}\right) \\
& =-\frac{\eta_{1}}{\omega_{1}}+\left(\frac{\pi}{2 \omega_{1}}\right)^{2}\left\{\frac{1}{\sin ^{2}\left(\frac{\pi u}{2 \omega_{1}}\right)}-8 \sum_{n=1}^{\infty} \frac{n q^{n}}{1-q^{n}} \cos 2 n\left(\frac{\pi u}{2 \omega_{1}}\right)\right\}, \quad q=e^{2 i \pi \tau},
\end{aligned}
$$

and according to 3.6 ,

$$
\frac{\eta_{1}}{\omega_{1}}=\frac{1}{3}\left(\frac{\pi}{2 \omega_{1}}\right)^{2}\left(1-24 \sum_{n \geq 1} \frac{n q^{n}}{1-q^{n}}\right)=\frac{1}{3}\left(\frac{\pi}{2 \omega_{1}}\right)^{2} E_{2}(\tau)
$$

We now solve our problem in the annulus $C_{R}$ rather in $A_{r}$, that is, for a fixed $Z \in C_{R}$ we solve

$$
\wp\left(\log (Z \bar{W}) ; 2 \omega_{1}, 2 \omega_{3}\right)+\frac{\eta_{1}}{\omega_{1}}=0
$$

in $W$ by using the methods in [2] and [3]. We introduce the new variable $u, 2 u \omega_{1}=$ $\log (Z \bar{W})$, and by the homogeneity properties of the $\wp$-function the equation (3.14) simply becomes

$$
\wp(u ; \tau)=\phi(\tau)
$$

where $\wp(u ; \tau)=\wp(u ; 1, \tau), \phi(\tau)=-\frac{\pi^{2}}{3} E_{2}(\tau)$. From the differential equation for the $\wp$-function, Eichler and Zagier obtained an expression of the second derivative $u^{\prime \prime}(\tau)$ of the solution of 3.15 :

$$
u^{\prime \prime}(\tau)=u_{\phi}^{\prime \prime}(\tau)= \pm \frac{1}{72 \pi^{2}}\left(4 \phi^{3}-g_{2} \phi-g_{3}\right)^{-\frac{3}{2}} \mathcal{E}(\tau)
$$

with

$$
\begin{aligned}
\mathcal{E}(\tau)= & -2\left(4 \phi^{3}-g_{2} \phi-g_{3}\right) \phi^{* *}+\left(12 \phi^{2}-g_{2}\right) \phi^{* 2}+\left(36 g_{3} \phi+2 g_{2}^{2}\right) \phi^{*} \\
& +12 g_{2} \phi^{4}+3 g_{2}^{2} \phi^{2}+6 g_{2} g_{3} \phi-g_{2}^{3}+27 g_{3}^{2}
\end{aligned}
$$

and

$$
\phi^{*}=12 \pi^{2}\left(\frac{1}{2 i \pi} \frac{d}{d \tau}-\frac{1}{6} E_{2}\right) \phi, \phi^{* *}=12 \pi^{2}\left(\frac{1}{2 i \pi} \frac{d}{d \tau}-\frac{1}{3} E_{2}\right) \phi^{*}
$$


It is known that the differential operator $\frac{1}{2 i \pi} \frac{d}{d \tau}-\frac{k}{12} E_{2}$ sends the space of modular forms of weight $k$ for some subgroup of $\mathrm{SL}_{2}(\mathbb{Z})$ into the space of modular forms of weight $k+2$, hence for $\phi(\tau)=e_{k}(\tau), \phi^{*}$ is a modular form of weight 4 and $\phi^{* *}(\tau)$ is a modular form of weight 6 for the subgroup $\Gamma(2)$ of $\mathrm{SL}_{2}(\mathbb{Z})$.

By using the fundamental fact [11, 16] that every modular form on $\Gamma$ is uniquely expressible as a polynomial in $E_{4}$ and $E_{6}$ and the extension $\mathbb{C}\left[E_{2}, E_{4}, E_{6}\right]$ of $\mathbb{C}\left[E_{4}, E_{6}\right]$ is closed under differentiation,

$$
\left\{\begin{array}{l}
\frac{1}{2 i \pi} \frac{d}{d \tau} E_{4}=\frac{1}{3}\left(E_{2} E_{4}-E_{6}\right), \\
\frac{1}{2 i \pi} \frac{d}{d \tau} E_{6}=\frac{1}{2}\left(E_{2} E_{6}-E_{4}^{2}\right), \\
\frac{1}{2 i \pi} \frac{d}{d \tau} E_{2}=\frac{1}{12}\left(E_{2}^{2}-E_{4}\right) .
\end{array}\right.
$$

Equation (3.16) becomes, after some calculations,

$u^{\prime \prime}(\tau)= \pm \frac{\sqrt{3}}{144} \pi \frac{-E_{2}^{6}+15 E_{2}^{4} E_{4}-40 E_{2}^{3} E_{6}+45 E_{2}^{2} E_{4}^{2}-24 E_{2} E_{4} E_{6}-27 E_{4}^{3}+32 E_{6}^{2}}{\left(3 E_{2} E_{4}-2 E_{6}-E_{2}^{3}\right)^{\frac{3}{2}}}$.

With the function $\Phi_{r, s}$ as given in (3.10), we use the following identities of Ramanujan [1]:

$$
1728 \Phi_{2,3}=-E_{2}^{3}+3 E_{2} E_{4}-2 E_{6}, \quad \Phi_{5,6}=\left(q \frac{d}{d q}\right) \Phi_{4,5}
$$

to obtain

$$
20736 \Phi_{5,6}=\left(q \frac{d}{d q}\right)\left(15 E_{2} E_{4}^{2}-20 E_{2}^{2} E_{6}+10 E_{2}^{3} E_{6}-4 E_{4} E_{6}-E_{2}^{5}\right) .
$$

Consequently, the previous derivatives formulas (3.19) give

$248832 \Phi_{5,6}=9 E_{4}^{3}+16 E_{6}^{2}+5\left(-E_{2}^{6}+15 E_{2}^{4} E_{4}-40 E_{2}^{3} E_{6}+45 E_{2}^{2} E_{4}^{2}-24 E_{2} E_{4} E_{6}\right)$.

Using the relation $1728 \Delta=E_{4}^{3}-E_{6}^{2}$, we deduce

$$
\begin{aligned}
u^{\prime \prime}(\tau) & = \pm \frac{1728 \sqrt{3}}{5} \pi \frac{\Phi_{5,6}(\tau)-\Delta(\tau)}{\Phi_{2,3}(\tau)^{\frac{3}{2}}} \\
& = \pm \frac{1728 \sqrt{3}}{5} \pi\left(120 e^{i \pi \tau}-1440 e^{3 i \pi \tau}+21600 e^{5 i \pi \tau}+\cdots\right) .
\end{aligned}
$$

Let us denote the constant $\frac{1728 \sqrt{3}}{5} \pi$ by $C_{0}$. If we integrate twice in the above expression for $u^{\prime \prime}$, we get

$$
u(\tau)=C_{1}+C_{2} \tau \pm C_{0} \int_{\tau}^{i \infty} \frac{\Phi_{5,6}(t)-\Delta(t)}{\Phi_{2,3}(t)^{\frac{3}{2}}}(t-\tau) d t,
$$

where the path of integration may be any curve which does not pass through a zero of $\Phi_{2,3}(t)$. For $\Im \tau>1$

$$
u(\tau)=C_{1}+C_{2} \tau \pm \frac{C_{0}}{\pi^{2}}\left(120 e^{i \pi \tau}-160 e^{3 i \pi \tau}+4320 e^{5 i \pi \tau}+\cdots\right) .
$$

The constants $C_{1}, C_{2}$ are determined by analyzing the behavior of $u(\tau)$ for $\tau$ near $\infty$. The equation (3.15) has the particular solution

$$
u_{0}(\tau)=-\int_{\infty}^{\phi(\tau)} \frac{d x}{2 \sqrt{\left(x-e_{1}\right)\left(x-e_{2}\right)\left(x-e_{3}\right)}} .
$$


The integral is on any path which does not contain any of the real zeros $e_{3}<e_{2}<e_{1}$ of $4 x^{3}-g_{2} x-g_{3}$. We would like to investigate the behavior of this integral when $\tau$ goes to $i \infty$. Making the substitution $z=\frac{x-e_{3}}{e_{2}-e_{3}}$, we obtain

$$
\begin{aligned}
& -\int_{\infty}^{\phi(\tau)} \frac{d x}{2 \sqrt{\left(x-e_{1}\right)\left(x-e_{2}\right)\left(x-e_{3}\right)}} \\
& \quad=-\frac{1}{\sqrt{e_{1}-e_{3}}} \int_{\infty}^{\frac{\phi(\tau)-e_{3}}{e_{2}-e_{3}}} \frac{d z}{2 \sqrt{z(1-z)(1-\lambda z)}} \\
& \quad=\frac{\tau}{2}-\frac{1}{\sqrt{e_{1}-e_{3}}} \int_{0}^{\frac{\phi(\tau)-e_{3}}{e_{2}-e_{3}}} \frac{d z}{2 \sqrt{z(1-z)(1-\lambda z)}}
\end{aligned}
$$

where $\lambda=\frac{e_{2}-e_{3}}{e_{1}-e_{3}}$ is the elliptic modular function. The importance of the above change of variables is that $\left|\lambda \frac{\phi(\tau)-e_{3}}{e_{2}-e_{3}}\right|<1$ for $|\tau|$ large. We will analyze the integral as an incomplete hypergeometric function. We expand $(1-\lambda z)^{-\frac{1}{2}}$ in power series and integrate term by term using the identity

$$
(n+1) \int_{0}^{x} z^{n+1} \frac{d z}{\sqrt{z(1-z)}}=\left(n+\frac{1}{2}\right) \int_{0}^{x} z^{n} \frac{d z}{\sqrt{z(1-z)}}-x^{n} \sqrt{x(1-x)} .
$$

We get with $y=\frac{\phi(\tau)-e_{3}}{e_{2}-e_{3}}$ :

$$
u_{0}(\tau)=\frac{\tau}{2}-\frac{a}{2 \sqrt{e_{1}-e_{3}}} \int_{0}^{y} \frac{d z}{\sqrt{z(1-z)}}-\sqrt{y(1-y)} \sum_{n \geq 0} a_{n} y^{n}
$$

where

$$
\begin{aligned}
a & =1+\sum_{m \geq 1}\left(\frac{1 \cdot 3 \cdots(2 m-1)}{2 \cdot 4 \cdots 2 m}\right)^{2} \lambda^{m} \\
a_{n} & =\sum_{m \geq n+1} \frac{1 \cdot 3 \cdots(2 m-1)}{2 \cdot 4 \cdots 2 m}\left(\frac{(2 n+3) \cdots(2 m-1)}{(2 n+2) \cdots 2 m}\right) \lambda^{m}
\end{aligned}
$$

We consider in $\widehat{\mathbb{C}} \backslash(-\infty, 0] \cup[1,+\infty)$ the branch of $\sqrt{y(1-y)}$ which is positive in $(0,1)$ and the branch of $\int_{0}^{z} \frac{d u}{\sqrt{u(1-u)}}$ given by

$$
\int_{0}^{z} \frac{d u}{\sqrt{u(1-u)}}=\frac{\pi}{2}+i \log (2 \sqrt{z(1-z)}-i(2 z-1)) .
$$

This corresponds to the choice of the branch of $\sin ^{-1} z=-i \log \left(\sqrt{1-z^{2}}+i z\right)$ which is a conformal map between $\mathbb{C} \backslash(-\infty,-1] \cup[1,+\infty)$ and $\left\{z \in \mathbb{C},|\Re z|<\frac{\pi}{2}\right\}$, so we may write

$$
u_{0}(\tau)=\frac{\tau}{2}-\frac{a}{2 \sqrt{e_{1}-e_{3}}}\left(\frac{\pi}{2}+i \log (2 \sqrt{y(1-y)}-i(2 y-1))\right)+\sqrt{y(1-y)} \sum_{n \geq 0} a_{n} y^{n} .
$$


The general value of $u(\tau)$ is $m+n \tau \pm u_{0}(\tau)$. The behavior of $u_{0}(\tau)$ as $\tau$ tends to $i \infty$ is a consequence of the following known identities:

$$
\begin{aligned}
& e_{1}=\phi(\tau)+\pi^{2}+8 \pi^{2} \sum_{n \geq 1} \frac{e^{2 i \pi n \tau}}{\left(1+e^{2 i \pi n \tau}\right)^{2}}, \\
& e_{2}=\phi(\tau)+8 \pi^{2} \sum_{n \geq 1} \frac{e^{i \pi(2 n-1) \tau}}{\left(1+e^{i \pi(2 n-1) \tau}\right)^{2}} \\
& e_{3}=\phi(\tau)-8 \pi^{2} \sum_{n \geq 1} \frac{e^{i \pi(2 n-1) \tau}}{\left(1-e^{i \pi(2 n-1) \tau}\right)^{2}}
\end{aligned}
$$

and the approximation $\lambda(\tau)=\frac{e_{2}-e_{3}}{e_{1}-e_{3}} \sim 16 e^{i \pi \tau}$. An easy computation yields, as $\tau$ tends to $i \infty$,

$$
u_{0}(\tau)=\frac{\tau}{2}-\frac{1}{4}+\mathrm{O}\left(e^{i \pi \tau}\right)
$$

It follows that $C_{1}=\frac{-1}{4}, C_{2}=\frac{1}{2}$.

The equality $\arg Z-\arg W(Z)=\pi$ is a consequence of the fact that for $\tau=\frac{i \pi}{\log R}$, the integral $\int_{\tau}^{i \infty} \frac{\Phi_{5,6}(t)-\Delta(t)}{\Phi_{2,3}(t)^{\frac{3}{2}}}(t-\tau) d t$ is real. We have thus obtained a precise analysis of the motion of the unique zero of the Bergman kernel of the annulus. By symmetry, we suppose that $Z=e^{\alpha}, 0<\alpha<\log R$, and we denote by $W_{R}$ the corresponding zero. We observe that

$$
\frac{3}{4}+\frac{1728 \sqrt{3}}{5} \pi \int_{\tau}^{i \infty} \frac{\Phi_{5,6}(t)-\Delta(t)}{\Phi_{2,3}(t)^{\frac{3}{2}}}(t-\tau) d t \notin\left\{0, \frac{1}{2}\right\}+\mathbb{Z},
$$

otherwise

$$
\wp\left(u_{0} ; \tau\right)=\phi(\tau)=e_{3}=\phi(\tau)-8 \pi^{2} \sum_{n \geq 1} \frac{e^{i \pi(2 n-1) \tau}}{\left(1-e^{i \pi(2 n-1) \tau}\right)^{2}}
$$

or

$$
\wp\left(u_{0} ; \tau\right)=\phi(\tau)=e_{2}=\phi(\tau)+8 \pi^{2} \sum_{n \geq 1} \frac{e^{i \pi(2 n-1) \tau}}{\left(1+e^{i \pi(2 n-1) \tau}\right)^{2}} .
$$

This is impossible for $\tau=i \pi / \log R$.

As usual, the fractional part $\langle x\rangle$ of $x \in \mathbb{R}$ is such that $0 \leq\langle x\rangle\langle 1, x-\langle x\rangle \in$ $\mathbb{Z}$. We denote by $\beta_{0}$ the fractional part of $\frac{3}{4}+\frac{1728 \sqrt{3}}{5} \pi \int_{\tau}^{i \infty} \frac{\Phi_{5,6}(t)-\Delta(t)}{\Phi_{2,3}(t)^{\frac{3}{2}}}(t-\tau) d t$. An easy computation shows that if $\beta_{0}<1 / 2$, then the extreme positions of the equilibrium points of the Green's function, $W_{1}, W_{R}$, or the zeros of the Bergman kernel for $Z=1$ and $Z=R$ are $W_{1}=-R^{2 \beta_{0}}, W_{R}=-R^{1-2 \beta_{0}}$. Whereas if $\beta_{0}>1 / 2, W_{1}=-R^{2-2 \beta_{0}}, W_{R}=-R^{2 \beta_{0}-1}$, with $W_{1} \cdot W_{R}=R$.

Remark 3.3. The same method with another change of variables can give another expression to the result of Eichler and Zagier for the solution of $\wp\left(z_{0}, \tau\right)=0$. In fact, we write a solution in the following form:

$$
z_{0}(\tau)=-\int_{0}^{\infty} \frac{d x}{2 \sqrt{\left(x-e_{1}\right)\left(x-e_{2}\right)\left(x-e_{3}\right)}},
$$

but here we make the substitution

$$
x-e_{3}=\frac{e_{1}-e_{3}}{z}, \quad z=\frac{e_{1}-e_{3}}{x-e_{3}}
$$


and use the fact that $\left|\lambda \frac{e_{3}-e_{2}}{e_{3}}\right|$ is very small for $|\tau|$ large. The same method gives with $y=\frac{e_{3}-e_{1}}{e_{3}}$

$$
z_{0}(\tau)=\frac{a}{2 \sqrt{e_{1}-e_{3}}} \int_{0}^{y} \frac{d z}{\sqrt{z(1-z)}}+\sqrt{y(1-y)} \sum_{n \geq 0} a_{n} y^{n} .
$$

The term $\frac{1}{2 \sqrt{e_{1}-e_{3}}} \int_{0}^{y} \frac{d z}{\sqrt{z(1-z)}}$ tends to $\pm \frac{1}{2} \pm \frac{\log (2 \sqrt{6}+5)}{2 i \pi}$ when $\tau$ tends to $i \infty$ as in [2].

\section{Some identities}

The constant $-\pi^{2} / 3$ in (3.15) seems very special, but actually it is very natural. It is classical (9, p. 415) that the Weierstrass sigma function associated to the periods $2 \omega_{1}$ and $2 \omega_{3}$ is related to the theta function $\theta_{1}(z, \tau)$ by

$$
\sigma\left(u, 2 \omega_{1}, 2 \omega_{3}\right)=2 \omega_{1} e^{2 \eta_{1} \omega_{1} v^{2}} \frac{\theta_{1}(v)}{\theta_{1}^{\prime}(0)}, \quad u=2 \omega_{1} v .
$$

Consequently the function $\Phi(v, \tau)=\sigma\left(u, 2 \omega_{1}, 2 \omega_{3}\right) e^{-2 \eta_{1} \omega_{1} v^{2}}$ admits the following infinite product decomposition (9, p. 424):

$$
\Phi(v, \tau)=\frac{-i \omega_{1}}{\pi}\left(z-z^{-1}\right) \prod_{n=1}^{\infty} \frac{\left(1-q^{n} z^{2}\right)\left(1-q^{n} z^{-2}\right)}{\left(1-q^{n}\right)^{2}}, \quad z=e^{i \pi v} .
$$

From (3.13), we have precisely

$$
\frac{d^{2}}{d v^{2}} \log \Phi(v, \tau)=-\wp(v, \tau)-\frac{\pi^{2}}{3} E_{2}(\tau)=-\sum_{n \in \mathbb{Z}} \frac{1}{\sinh \left(\frac{i n \pi \tau+v}{2}\right)^{2}} .
$$

These functions are studied from a very different point of view in 8 . Our result gives the zero of the second derivative $\frac{d^{2}}{d v^{2}} \log \Phi(v, \tau)$.

For $k \geq 4$, the zeros of Eisenstein series $E_{k}$ have interested several authors, but to our knowledge no result is available for the zeros of the series $E_{2}$ (or the critical points of the discriminant function $\Delta(t)$ ). As a function of the real variable $0 \leq q<1$, the series $1-24 \sum_{n=1}^{\infty} \sigma_{1}(n) q^{n}$ is a decreasing function from 1 to $-\infty$, hence it has a unique zero $q_{0}$ in $(0,1)$ corresponding to a pure imaginary number $z_{0}=i \gamma_{0}, \gamma_{0} \in \mathbb{R}$ of the Eisenstein series $E_{2}(\tau)$. According to D. Zagier, there are infinitely many zeros of $E_{2}(\tau)$ in the half-strip $\{0 \leq \Im \tau<1\}$ (private communication, via H. Cohen). The following two zeros were computed for us by H. Cohen:

$$
\begin{gathered}
z_{0}=0.52352170001799926680053440480610976968 \ldots i, \\
z_{1}=\frac{1}{2}+0.13091903039676244690411482601971302060 \ldots i
\end{gathered}
$$

with $z_{0}=\frac{\log q_{0}}{2 i \pi}$. By comparing the formulas in Theorem 3.2 and in 2] (corollary) when $\tau$ is a zero of the series $E_{2}$, we obtain some identities. In particular

Corollary 4.1. Let $z_{0}=i \gamma_{0}$ be a zero of the Eisenstein series $E_{2}, \gamma_{0} \in \mathbb{R}$. Then $\gamma_{0}$ verifies the two integral equations with some integers $n$ and $m$ :

$$
\frac{(2 n+1) \gamma_{0} \pi-\log (5+2 \sqrt{6})}{288 \pi^{2} \sqrt{6}}=\int_{\gamma_{0}}^{\infty} \frac{\Delta(i t)}{E_{6}(i t)^{\frac{3}{2}}}\left(t-\gamma_{0}\right) d t
$$


and

$$
\frac{1}{4}+m=\frac{1728 \sqrt{3}}{5} \pi \int_{\gamma_{0}}^{\infty} \frac{\Phi_{5,6}(i t)-\Delta(i t)}{\Phi_{2,3}(i t)^{\frac{3}{2}}}\left(t-\gamma_{0}\right) d t .
$$

We observe that from its infinite product expansion, $\Delta(t)$ does not vanish on the upper half plane and moreover the series $E_{6}(\tau)$ has on the imaginary axis a unique zero at $i$. The integral in the preceding corollary must be taken first on a segment $\left[i \gamma_{0}, i-i \epsilon\right]$ followed by the half circle centered at $i$, of radius $\epsilon$ and finally on $[i+i \epsilon, i \infty)$.

We refer to 3 for the complete definition of Jacobi forms. These are holomorphic functions $\Phi: \mathcal{H} \times \mathbb{C} \mapsto \mathbb{C}$ such that

(1) $\Phi\left(\frac{a \tau+b}{c \tau+d}, \frac{z}{c \tau+d}\right)=(c \tau+d)^{k} e^{\frac{2 i \pi m z^{2}}{c \tau+d}}, \quad\left(\left(\begin{array}{cc}\alpha & \beta \\ \gamma & \delta\end{array}\right) \in \mathrm{SL}_{2}(\mathbb{Z})\right)$,

(2) $\Phi(\tau, z+\lambda \tau+\mu)=e^{-2 i \pi m\left(\lambda^{2} \tau+2 \lambda z\right)} \Phi(\tau, z), \quad(\lambda, \mu) \in \mathbb{Z}^{2}$,

(3) $\Phi(\tau, z)$ has a Fourier expansion of the form

$$
\Phi(\tau, z)=\sum_{n=0}^{\infty} \sum_{r \in \mathbb{Z}, r^{2} \leq 4 n m} c(n, r) e^{2 i \pi(n \tau+r z)},
$$

$k$ and $m$ are natural numbers called the weight and index of $\Phi$. The ring of Jacobi forms of index 1 is generated by the Jacobi-Eisenstein series $E_{4,1}$ of weight 4 and $E_{6,1}$ of weight 6 [3] (p. 23):

$$
\begin{gathered}
E_{4,1}=1+\left(\zeta^{2}+56 \zeta+126+56 \zeta^{-1}+\zeta^{-2}\right) q+\cdots, \\
E_{6,1}=1+\left(\zeta^{2}-88 \zeta-330-88 \zeta^{-1}+\zeta^{-2}\right) q+\cdots
\end{gathered}
$$

with $q=e^{2 i \pi \tau}, \zeta=e^{2 i \pi z}$ and

$$
\begin{aligned}
& \Phi_{10,1}=\frac{1}{144}\left(E_{6} E_{4,1}-E_{4} E_{6,1}\right), \\
& \Phi_{12,1}=\frac{1}{144}\left(E_{4}^{2} E_{4,1}-E_{6} E_{6,1}\right) .
\end{aligned}
$$

By Theorem 3.6 of [3], $\frac{\Phi_{12,1}(\tau, z)}{\Phi_{10,1}(\tau, z)}=-\frac{3}{\pi^{2}} \wp(\tau, z)$. By (3.19), if $z=z(\tau)$ is the solution of $\wp(z, \tau)=-\frac{\pi^{2}}{3} E_{2}$ as given in Theorem 3.2, we have $3 E_{4}^{\prime} E_{6,1}(z, \tau)=2 E_{6}^{\prime} E_{4,1}(z, \tau)$.

\section{LOCATION AND MOTIONS OF THE EQUILIBRIUM POINT}

We now give the result on the location of the equilibrium point for the Green's function.

Theorem 5.1. For each $R>1$ and for each $z \in C_{R}=\{1<|z|<R\}$, the Green's function $G(z, w)$ has a unique equilibrium point $w=F(z)$ located on the same diameter as $z$ and that satisfies the inequalities

$$
\begin{array}{ll}
-|z| \leq-|w| \leq-\sqrt{R}, & \sqrt{R} \leq|z| \leq R, \\
-\sqrt{R} \leq-|w| \leq-|z|, & 1 \leq|z| \leq \sqrt{R} .
\end{array}
$$

Furthermore, the function $F(z)$ approaches at the boundary the zero $w(z)$ of the Bergman kernel. If $z=e^{\alpha}$ is real, this zero $w=e^{u}$ is given by the equation

$$
\wp\left(u ; 2 \omega_{1}, 2 \omega_{3}\right)=\wp\left(\alpha ; 2 \omega_{1}, 2 \omega_{3}\right)+\frac{\wp^{\prime}\left(\alpha ; 2 \omega_{1}, 2 \omega_{3}\right)}{2 \zeta\left(\alpha ; 2 \omega_{1}, 2 \omega_{3}\right)-2 \lambda \alpha} .
$$


Proof. The two inequalities between $z$ and $w=F(z)$ are proved in [12] (p. 64). Without loss of generality, we suppose from the start that $z=e^{\alpha}$ is real so that $0<\alpha<\log R$. The Green's function of $C_{R}$ with pole at $z$ has the following form, derived from (2.8):

$$
G(z, w)=\Re V(\log z), \quad V(u)=\frac{\sigma(u+\alpha)}{\sigma(u-\alpha)} e^{-2 \lambda \alpha u},
$$

where $\sigma$ is the Weierstrass sigma function associated to the periods $\omega_{1}=\log R$, $\omega_{3}=i \pi$ and $\lambda=\frac{\eta_{1}}{\omega_{1}}=\frac{1}{3}\left(\frac{\pi}{2 \omega_{1}}\right)^{2} E_{2}(\tau)$. The equilibrium point $w=e^{u}$ for $G(z, w)$ is given by the equation

$$
\zeta(u+\alpha)-\zeta(u-\alpha)=2 \alpha \lambda=2 \alpha \frac{1}{3}\left(\frac{\pi}{2 \omega_{1}}\right)^{2} E_{2}(\tau) .
$$

As is shown in 10, there is a solution corresponding to the unique equilibrium point in the annulus $C_{R}$. By elementary transformations, this equation is equivalent to

$$
\wp\left(u ; 2 \omega_{1}, 2 \omega_{3}\right)=\wp\left(\alpha ; 2 \omega_{1}, 2 \omega_{3}\right)+\frac{\wp^{\prime}\left(\alpha ; 2 \omega_{1}, 2 \omega_{3}\right)}{2 \zeta\left(\alpha ; 2 \omega_{1}, 2 \omega_{3}\right)-2 \lambda \alpha}
$$

hence it is of the type considered before, but of a more complicated nature. We would like to point out that (5.1) must be in accordance with the Schiffer relation (3.1) $K(z, \bar{t})=-\frac{2}{\pi} \frac{\partial^{2} G(z, t)}{\partial z \partial t}$. For a real $z=x_{0} \in C_{R}$, the equilibrium point $w=x$ is also real, and the limiting position of $x$ when $x_{0}$ tends to the boundary of $C_{R}$ is given by $\frac{d x}{d x_{0}}=0$. We simply denote $F\left(x, x_{0}\right)=\frac{\partial G\left(x, x_{0}\right)}{\partial x}$ so that

$$
\frac{d x}{d x_{0}}=-\frac{\frac{\partial F\left(x, x_{0}\right)}{\partial x_{0}}}{\frac{\partial F\left(x, x_{0}\right)}{\partial x}}=-\frac{\frac{\partial^{2} G\left(x, x_{0}\right)}{\partial x \partial x_{0}}}{\frac{\partial^{2} G\left(x, x_{0}\right)}{\partial^{2} x}},
$$

and $\frac{d x}{d x_{0}}=0$ if and only if

$$
\frac{\partial F\left(x, x_{0}\right)}{\partial x_{0}}=\frac{\partial^{2} G\left(x, x_{0}\right)}{\partial x \partial x_{0}}=0 .
$$

According to the Schiffer relation and (3.3), we obtain $\frac{d x}{d x_{0}}=0$ if and only if $K\left(x_{0}, x\right)=0$. This explains the relation between the limiting positions of the equilibrium points and the zeros of the Bergman kernel. It is also possible to interpret (5.1) as a perturbed form of (3.15). We use the classical formula giving the functions $\wp$ and $\zeta$ in terms of theta function with $z=\frac{\pi \alpha}{2 \omega_{1}}$ as follows:

$$
\begin{aligned}
\left.\zeta\left(\alpha ; \omega_{1}, \omega_{3}\right)\right) & =\frac{\eta_{1}}{\omega_{1}} \alpha+\frac{\pi}{2 \omega_{1}} \frac{d}{d z} \ln \theta\left[\begin{array}{l}
1 \\
1
\end{array}\right](z, \tau) \\
\wp\left(\alpha ; 2 \omega_{1}, 2 \omega_{3}\right) & =-\frac{\eta_{1}}{\omega_{1}}-\left(\frac{\pi}{4 \omega_{1}}\right)^{2} \frac{d^{2}}{d z^{2}} \ln \theta\left[\begin{array}{l}
1 \\
1
\end{array}\right](z, \tau) .
\end{aligned}
$$


The equation (5.1) becomes

$$
\wp\left(u ; 2 \omega_{1}, 2 \omega_{3}\right)=-\frac{\eta_{1}}{\omega_{1}}+\mathcal{F}(\alpha ; \tau)
$$

where a perturbative term

$$
\mathcal{F}(\alpha ; \tau)=-\left(\frac{\pi}{4 \omega_{1}}\right)^{2} \frac{d^{2}}{d z^{2}} \ln \theta\left[\begin{array}{l}
1 \\
1
\end{array}\right](z, \tau)-\frac{1}{2} \frac{\left(\frac{\pi}{4 \omega_{1}}\right)^{2} \frac{d^{3}}{d z^{3}} \ln \theta\left[\begin{array}{l}
1 \\
1
\end{array}\right](z, \tau)}{\frac{d}{d z} \ln \theta\left[\begin{array}{l}
1 \\
1
\end{array}\right](z, \tau)}
$$

Remark 5.2. The same analysis can be done for the equilibrium point of the Green's function of a domain bounded by two non-concentric circles $\mathcal{C}_{1}, \mathcal{C}_{2}$, the first being inside the second. For a given $z$ in this domain, there is a unique circle $\mathcal{C}$ through $z$ orthogonal to the boundary circles. The equilibrium point $w$ is on this circle.

\section{REFERENCES}

1. N.I.Achieser. Theory of Approximation, Dover Publications, Inc, New York, 1992. MR1217081 (94b:01041)

2. M.Eichler; D. Zagier. On the zeros of the Weierstrass $\wp$-function, Math. Ann. $258(1981 / 82)$, 399-407. MR0650945 (83e:10031)

3. M.Eichler; D. Zagier. The theory of Jacobi forms, Progress in Mathematics, vol. 55, Birkhäuser Boston, Inc., Boston, MA, 1985. MR0781735 (86j:11043)

4. Th. Falliero; A. Sebbar. Capacité de la réunion de trois intervalles et fonctions thêta de genre 2, J. Math. Pures Appl. 80 (2001), 409-443. MR.1832165 (2002f:31004)

5. J. Fay. Theta functions on Riemann surfaces, Lectures Notes in Math., vol. 352, SpringerVerlag, 1973. MR0335789 (49:569)

6. D.A. Hejhal. Theta functions, kernel functions, and Abelian integrals, Mem. Amer. Math. Soc., vol. 129, American Mathematical Society, Providence, R.I., 1972. MR0372187|(51:8403)

7. D.A. Hejhal. Some remarks on kernel functions and Abelian differentials, Arch. Rational Mech. Anal. 52 (1973), 199-204. MR0344453(49:9192)

8. F. Hirzebruch; Th. Berger; R. Jung. Manifolds and Modular Forms, Aspect of Mathematics. A publication of the Max-Planck-Institut für Mathematik, Bonn, 1992. MR1189136 (94d:57001)

9. C. Jordan. Cours d'Analyse de l'École Polytechnique, seconde partie. Chapitre VI, Fonctions elliptiques, Gauthiers-Villars, Paris, 1894.

10. A.J. Maria. Concerning the equilibrium point of Green's function for an annulus, Duke Math. J. 1 (1935), 491-495.

11. S. Ramanujan. On certain arithmetical functions, Trans. Cambridge Philos. Soc. 22 (1916), 159-184.

12. W. Rudin. Analytic functions of class $H_{p}$, Trans. Amer. Math. Soc. 78 (1955), 46-66. MR0067993 (16:810b)

13. M. Schiffer. The kernel function of an orthogonal system, Duke Math. J. 13 (1946), 529-540. MR0019115 (8:371a)

14. M.Schiffer; N.S Hawley. Connections and conformal mappings, Acta Math. 107 (1962), 175274 .

15. N. Suita; A. Yamada. On the Lu Qi-Keng conjecture, Proc. Amer. Math. Soc. 59 (1976), 222-224. MR0425185 (54:13142)

16. B. van der Pol. On a non-linear partial differential equation satisfied by the logarithm of the jacobian theta-functions, with arithmetical applications. I, II, Nederl. Akad. Wetensch. Proc., Ser. A 54 (1981) 261-271, 272-284 = Indag. Math. 13 (1951). MR0042599(13:135a) 
17. H. Widom. Extremal polynomials associated with a system of curves in the complex plane. Advances in Math. 3 (1969), 127-232. MR0239059 (39:418)

18. K. Zarankiewicz. Über ein numerisches Verfahren zur konformen Abbildung zweifach zusammenhängender Gebiete., Zeit. f. ang. Math. u. Mech. 14 (1934) 97-104.

LABAG, Laboratoire Bordelais D'Analyse et Géométrie, Institut de Mathématiques, Université Bordeaux I, 33405 Talence, France

E-mail address: $\quad$ sebbar@math.u-bordeaux.fr

Faculté des Sciences, Université d'Avignon, 84000 Avignon, France

E-mail address: Therese.Falliero@univ-avignon.fr 